\title{
TOWARD AN EMBEDDED SYSTEM FOR GESTURE RECOGNITION BASED ON ARTIFICIAL NEURAL NETWORK USING RECONFIGURABLE TARGET (CASE STUDY AND REVIEW)
}

\author{
Ahmad Reda \\ PhD student, Institute of Automation and Infocommunication, University of Miskolc, Hungary \\ 3515 Miskolc, Egyetemváros, Hungary, e-mail: autareda@uni-miskolc.hu \\ Tareq Alshoufi \\ MSc student, Institute of Automation and Infocommunication, University of Miskolc, Hungary \\ 3515 Miskolc, Egyetemváros, Hungary, e-mail: alshoufi@iit.uni-miskolc.hu

\section{Ahmed Bouzid} \\ assistant professor, Institute of Automation and Infocommunication, University of Miskolc, Hungary \\ 3515 Miskolc, Egyetemváros, Hungary, e-mail: qgebouzid@uni-miskolc.hu

\section{József Vásárhelyi} \\ associate professor, Institute of Automation and Infocommunication, University of Miskolc, Hungary \\ 3515 Miskolc, Egyetemváros, Hungary, e-mail: vajo@uni-miskolc.hu
}

\begin{abstract}
With a view to create an intelligent remote control for robot movements, this article treats the study case of dataset creation using RSG (Reference Signal Generator). Using artificial intelligence, the device recognizes the gestures of an operator. Indeed, a neural network can classify time series data coming from accelerometers, and for a beginning 4 gestures are taken into consideration. The most challenging work is to build a reference dataset that is necessary for the learning process. To train the neural network, a huge amount of reference data should be created (hundreds of thousands of time-series vectors per gesture per sensor), which cannot be done manually by an operator. To overcome the issue, an RSG is created. This article also describes how a 1-DoF arm has been designed to emulate the behavior of the human arm doing gestures as well as the data acquisition system. The system is based on a software/hardware co-design implemented on Programmable System on Chip (PSoC).
\end{abstract}

Keywords: Gesture Recognition, HW/SW co-design, Data Acquisition, Motor Control, IMU

\section{Nomenclature}

\begin{tabular}{|c|c|c|c|}
\hline ADC & Analogue to Digital Converter & PSoC & Programmable System on Chip \\
\hline ANN & Artificial Neural Network & RSG & Reference Signal Generator \\
\hline FPGA & Field Programmable Gate Array & $\mathrm{SoC}$ & System on Chip \\
\hline FPAA & Field Programmable Analog Array & 1-DoF & One Degree of Freedom \\
\hline $\begin{array}{l}\text { IDE } \\
\text { IoT }\end{array}$ & $\begin{array}{l}\text { Integrated Development Environment } \\
\text { Internet of Things }\end{array}$ & $\Theta_{\mathrm{A}}$ & $\begin{array}{l}\text { angle with the perpendicular axis to } \\
\text { the table }\end{array}$ \\
\hline $\begin{array}{l}\text { IMU } \\
\text { IP }\end{array}$ & $\begin{array}{l}\text { Inertial Measurement Unit } \\
\text { Intellectual Property }\end{array}$ & $\Theta_{\text {в }}$ & $\begin{array}{l}\text { angle that separates the stick with the } \\
\text { perpendicular axis to the table }\end{array}$ \\
\hline
\end{tabular}




\section{Introduction}

Reconfigurability is one of the most important advances on computing technologies. It provides high flexibility and the ability to dynamically reconfigure the architecture of the computing system. Programmable analogue circuits suffer from a lack of competitivity since the market is mainly held by Anadigm, which provides FPAAs (Field Programmable Analog Array) [1], [2]. Mixed-signals computing become popular since it is possible to manipulate analog, digital, and processing systems in the same chip, also known as SoC (System on Chip). However, the computing world in general is affected by productivity gap issue where the chip complexity (measured by the number of transistors per chip) is increasing faster than the productivity (measured by the number of transistors per staff-months). Design with reusable IP (Intellectual Property) is intended to reduce the productivity gap and by the way to democratize programmable system on chips [3], [4]. PSoC (Programmable System on Chip) by Cypress Semiconductor holds an important place on the market share of embedded in general, and the leader of some highgrowth markets like Wi-Fi/Bluetooth combo chips for Internet of Things (IoT) devices and SRAM memory chips [5]. The integrated development environment (IDE) used for PSoC designs (PSoC Creator) is indeed ergonomic and suitable for fast prototyping, in contrast the lack of simulation tool makes it less appropriate for complex designs [6]. In recent years Artificial Neural Network (ANN) has been growing to address the challenges of the computational complexity, time, power and resources consuming. In addition, ANN achieved a remarkable performance, and it has been used in many applications related to a variety of industries such as, motion planning mobile robots and image classification [7]. In this context, the article presents a Reference Signal Generator (RSG) for the purpose of training an ANN for gesture recognition [8], [9]. The document is divided into sections where it is about describing the issue and the constraints, then details about how the solution is designed and implemented. Finally, results are exposed then discussed.

\section{Issue and Proposed Solution}

In the first instance, a dataset has been manually created for 4 gestures (top-down, bottom-up, right-toleft, and left-to-right) with 4 tests and 2 speeds per gesture using an IMU of 3 accelerometers and 3 gyroscopes. The dataset has been enriched by transforming every signal using its time derivative, integration and some moving averages. That resulted in 768 reference signals, consequently, 192 reference signal per gesture. Figure 1 shows the trace of an acquired accelerometer data during the "Down" gesture (top-down movement). After training the Artificial Neural Network (ANN), unsatisfactory results have been obtained. The dataset should be supplemented with a high number of reference signals. It is a tedious task to repeat and record a gesture tens of thousands of times by hand. Here comes the need to create a Reference Signal Generator (RSG) that can build a huge dataset automatically. The idea is to implement a 1-DoF (degree of freedom) arm that can imitate the gesture.

\section{Design and Instrumentation}

The automated system is divided into two major parts that are the embedded system and the mechanism that are illustrated in Figure 2.

\subsection{The Mechanism}

The mechanical part of the automated system consists of a bipolar stepper motor with a stepping resolution angle of $1.8^{\circ}$ joined on its rotor to a stick that holds the IMU on the top. 
For generating the "Down" gesture, the motor is placed on a table on its side then the starting point of the stick follows the rule:

$$
\theta_{\mathrm{A}} \in\left[0,60^{\circ}\right]
$$

where $\theta_{A}$ represents the angle with the perpendicular axis to the table.

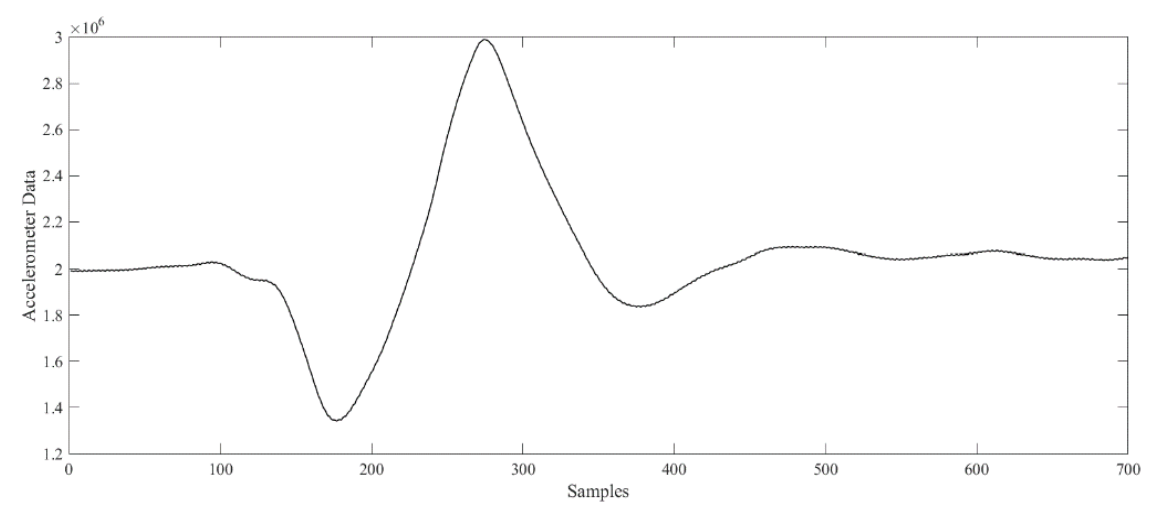

Figure 1. Target accelerometric signal of top-down gesture (reversed axis)

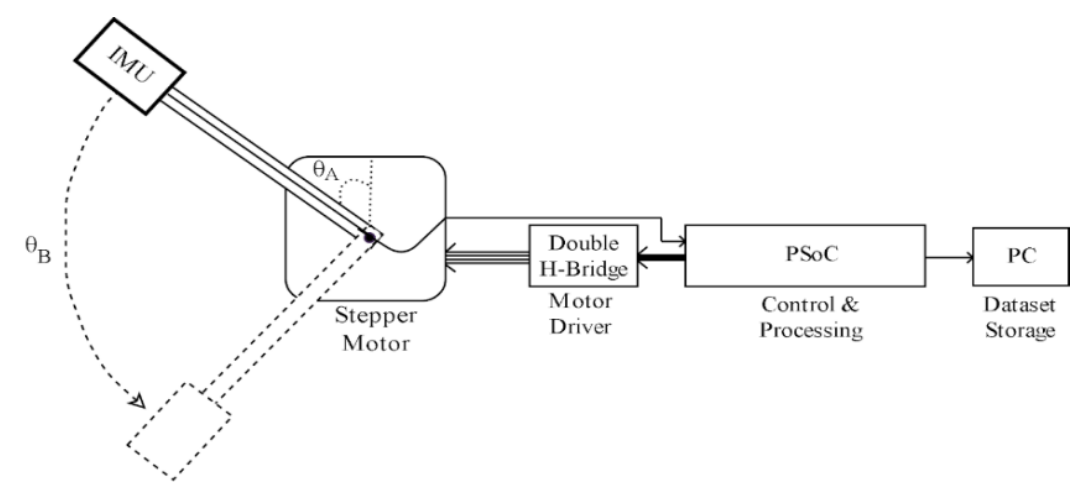

Figure 2. Functional schematic of the automated RSG

The ending point of the movement follows the rule:

$$
\theta_{\mathrm{B}} \in\left[\theta_{\mathrm{A}}+30^{\circ}, \theta_{\mathrm{A}}+60^{\circ}\right]
$$

where $\theta_{B}$ is also an angle that separates the stick with the perpendicular axis to the table. The combination of these angles can generate 162 signals.

\subsection{The Embedded System}

\subsubsection{The Process}

The first design was based on a single PSoC taking the advantage of the parallel processing, i.e. the digital part serves as hardware accelerator dedicated for stepper motor control (see Figure 3) and the software for data acquisition/transmission. Due to the absence of simulation tools and unpredictable behavior of the implementation, mainly originated from a non-negligible delay caused by the hardwareto-software and software-to-hardware data transfer, this derived to split the design into two PSoCs, one 
for stepper motor control (implemented on cy8ckit-050) and the other for sensor data acquisition/transmission (implemented on cy8ckit-059).

The embedded system performs the following tasks:

- The main process starts by setting the desired targets: $\theta_{A}$ and $\theta_{B}$ angles which are respectively the starting and ending angles of the motor movement with respect to the vertical axis.

- Setting the speed of the motor.

- Acquisition of sensors data then activation of the arm movement by enabling the hardware accelerator for motor control.

- All along the arm movement, from angle $\theta_{\mathrm{A}}$ to $\theta_{\mathrm{B}}$, the PSoC collects sensor data then transmits to PC via UART.

- When the motor reaches the desired $\theta_{\mathrm{B}}$ angle, the movement stops.

- Stop acquisition and data transmission a brief moment after stopping the movement (in order to collect some pseudo-periodic oscillations).

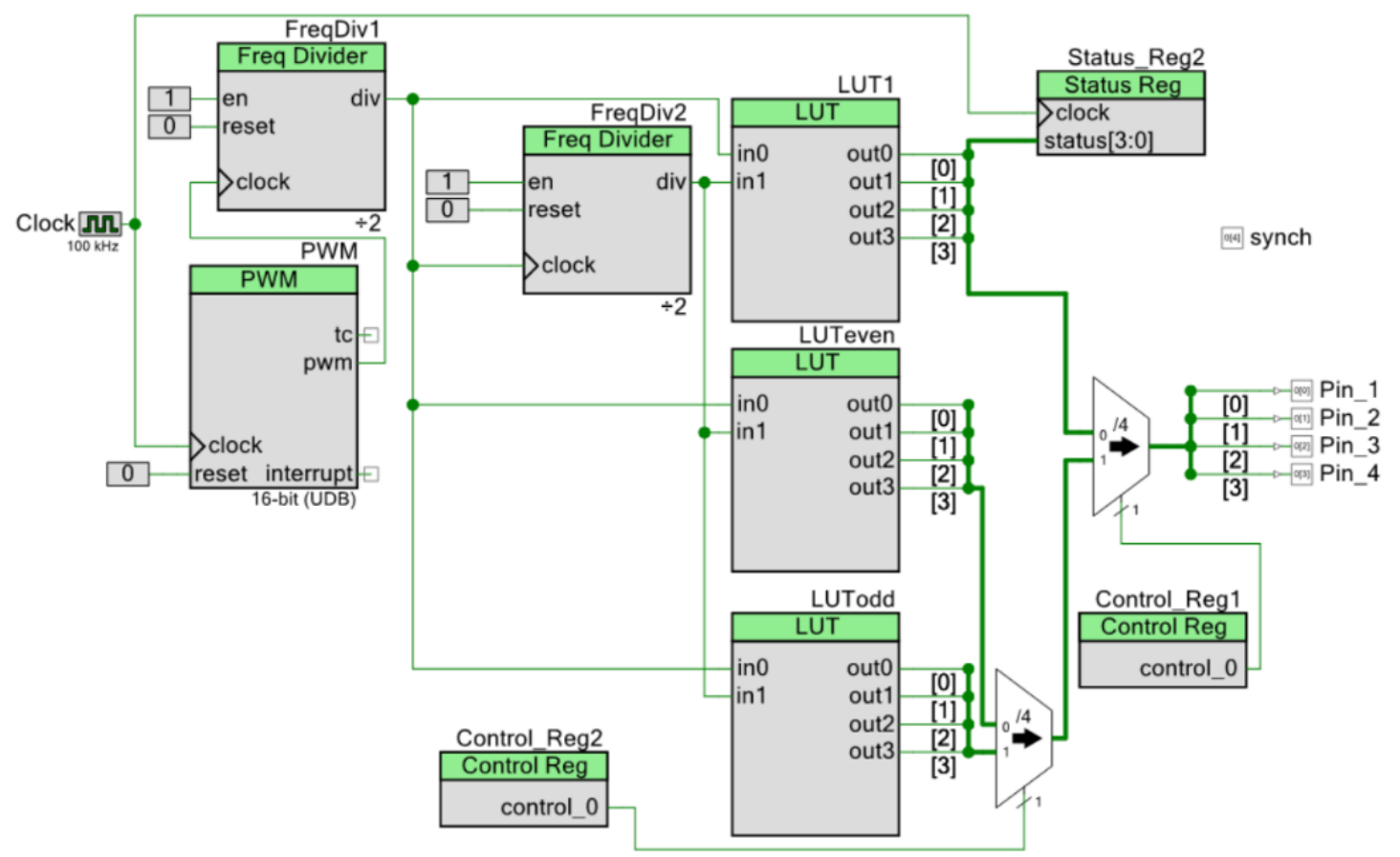

Figure 3. Schematic of the hardware accelerator dedicated to stepper motor control implemented on PSoC

\subsubsection{Data Acquisition/Transmission}

An ADC is used in the design that converts to 12 bits, so that the data are stored on 2 bytes. Conversion rate is set to 100000 samples per second (100 ksps). If the UART is set to $921600 \mathrm{bps}$, and the configuration is set to $8 / \mathrm{N} / 1$ ( 10 bits per frame, hence 20 bits per sample). Consequently, the transmission rate will be $46.06 \mathrm{ksps}$ which is less than the conversation rate which means that decreasing the conversion rate until $46.06 \mathrm{ksps}$ will not affect the transmission rate. The accelerometer used is ADXL335 analog accelerometer mounted on a GY-61 board equipped with a 3.3V regulator. Figure 4 shows the photography of the automated RSG. 


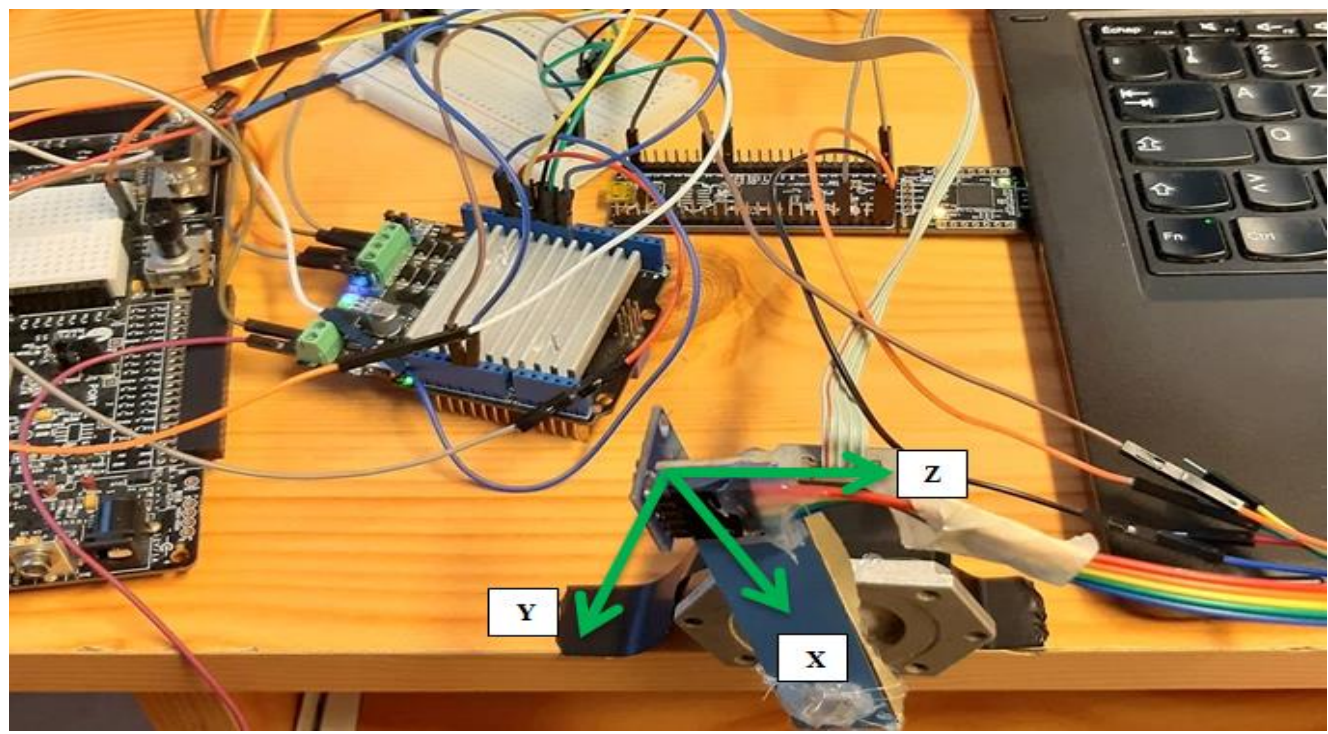

Figure 4. Photography of the automated RSG

\section{Results, Observations and Discussion}

The first experiment consisted of collecting acceleration data according to the $\mathrm{X}$ axis for the top-down gesture performed by hand traveling of around $45^{\circ}$. On this test the $\mathrm{X}$ axis was pointed to the top, and then Figure 1 shows the collected data. Figure 5 and Figure 6 are the results of the acquisition made by the automated system according to the $X$ axis for the bottom-up gesture traveling 14 degrees. Figure 6 was obtained by collecting accelerometric data of the automated system doing the top-down gesture for an angle travel of $12^{\circ}$.

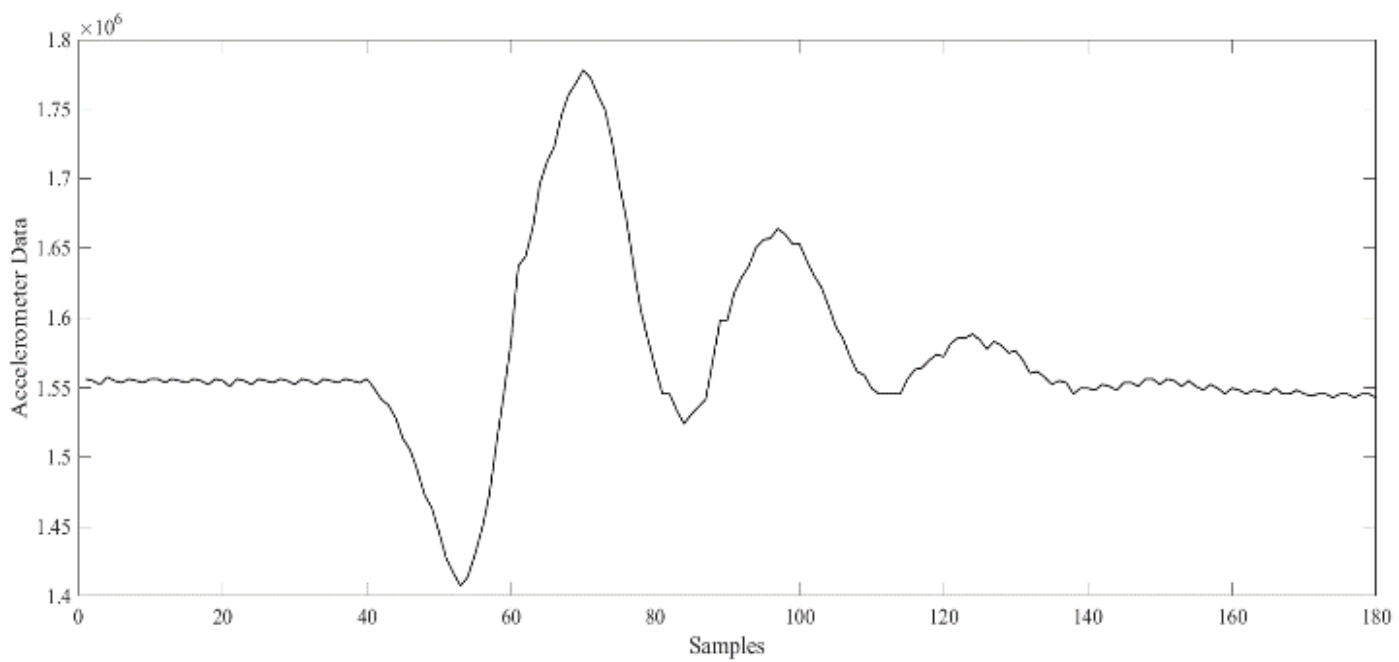

Figure 5. Plot of the data collected from the automated system for the bottom-up gesture 


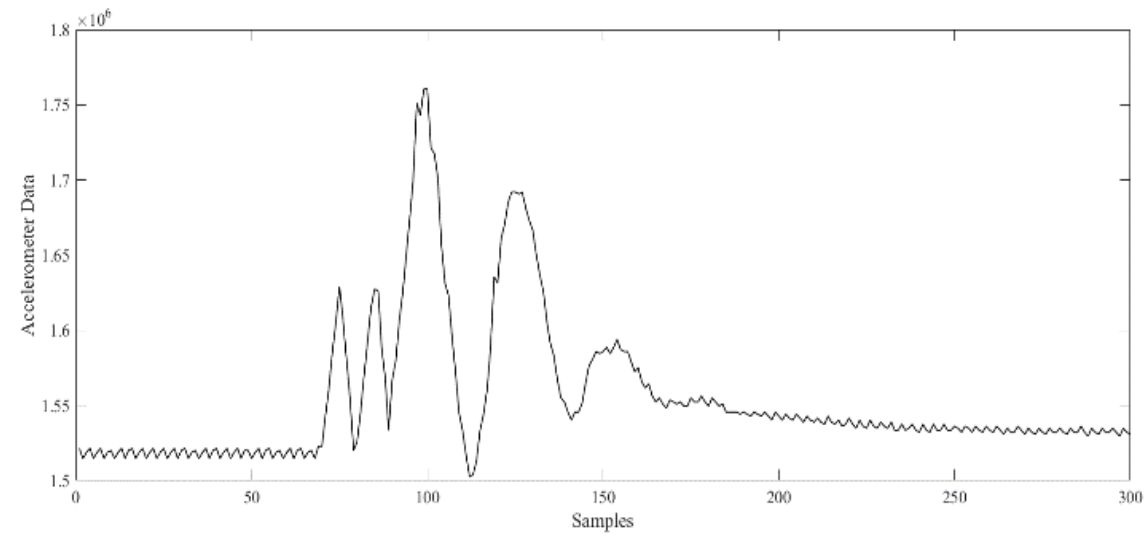

Figure 6. Plot of the data collected from the automated system for the top-down gesture.

Except for the manual movement, undesirable fluctuations in form of peaks are highly observed during the analysis of the plots. The two first peaks observed in Figure 6 are considered as noise because they do not appear on the desired signals generated by hand movements. These two peaks are mainly caused by the mechanical vibrations of the motor. The motor vibrations are due to the absence of required torque to handle the desired movement of the stick. Mainly, because of the limitation of the motor drive (Double H Bridge L298), which provides only 2 A. It is also visible on Figure 3 that the wires prevent obtaining correct signal due to their vibrations.

A hysteresis phenomenon is also observed on Figure 1, Figure 5 and Figure 6 due to the low quality of the sensor. The two last peaks (that appear after the mean peak), observed in Figure 5 and Figure 6, are considered as undesirable pseudo-periodic oscillations which are more intense than the ones observed on the manually created dataset. These two peaks reveal that the damping coefficient is much lower than the desired one. Here the mechanical system has an under damped response, thus needs to be redesigned for better damping. Table 1 resumes the hardware resources consumed by the hardware accelerator implemented on PSoC

Table1. Hardware resources consumed by the PSoC based hardware accelerator

\begin{tabular}{|c|c|c|}
\hline & Resources & Values \\
\hline \multirow{2}{*}{ System } & Digital Clocks & $1 / 8$ \\
\hline & Inputs and Outputs & $8 / 72$ \\
\hline \multirow{5}{*}{$\begin{array}{l}\text { UDB } \\
\text { (Univer- } \\
\text { sal } \\
\text { Digital } \\
\text { Block) }\end{array}$} & Macrocells & $18 / 192$ \\
\hline & Unique P-terms & $32 / 384$ \\
\hline & Datapath Cells & $2 / 24$ \\
\hline & Status Cells & $2 / 24$ \\
\hline & Control Cells & $3 / 24$ \\
\hline \multirow{2}{*}{ Memory } & Flash & $0.8 \%$ \\
\hline & SRAM & $4.3 \%$ \\
\hline
\end{tabular}




\section{Conclusion and perspective}

An embedded system has been designed and implemented for an automated Reference Signal Generator that leads to generate 174960 reference signals. PSoC provides the ability to use parallel processing for motor control and data acquisition/transmission but owing to the lack of simulation tools, which was not easy to create a full hardware accelerator for motor control.

The results show that the mechanical and the electrical systems should be reconsidered, by using a better motor driver, to obtain higher motor torque then less vibrations and higher damping hence better reference signals. The observed hysteresis leads us to consider sensor calibration for the next work and examine the possibility of creating an Artificial Reference Data Generator based on the mathematical model and simulation of the desired signals.

\section{Acknowledgment}

This research was supported by the European Union and the Hungarian State, co-financed by the European Regional Development Fund in the framework of the GINOP-2.3.4-15-2016-00004 project, aimed to promote the cooperation between the higher education and the industry.

\section{References}

[1] Györök, G., Orosz, T., Makó, M. and Treiber, T.: To Achieve Circuit Robustness by Co-operation of FPAA and Embedded Microcontroller. Timisoara, Romania, IEEE 8th International Symposium on Applied Computational Intelligence and Informatics (SACI), 2013, pp. 315-320. https://doi.org/10.1109/SACI.2013.6608989

[2] Wawryn, K., Suszynski, R.: Prototyping of WTA ANNs using FPAA devices. Bydgoszcz, Poland, 24th International Conference Mixed Design of Integrated Circuits and Systems, 2017, pp. 463467. https://doi.org/10.23919/MIXDES.2017.8005254

[3] Bouzid, A., Boucetta, S. I. and Vásárhelyi, J.: The PSoC 5 LP LABBOOK. Saarbrücken, Germany, Éditions Universitaires Européennes, ISBN: 978-613-8-43692-8, 2018. 72 p.

[4] Bouzid, A. and Vásárhelyi, J.: Survey About the PSOC5 LABBOOK. Lecture Notes in Mechanical Engineering, 2018, pp. 519-525. https://doi.org/10.1007/978-3-319-75677-6_45

[5] Sun, L.: 10 Reasons to Buy Cypress Semiconductor Stock Today. fool.com/investing/2018/02/11/10-reasons-to-buy-cypress-semiconductor-stock-toda.aspx. Accessed 06 Apr. 2019.

[6] Sgambelluri, N., et al.: An Artificial Neural Network approach for Haptic Discrimination in Minimally Invasive Surgery, Jeju, Korea, The 16th IEEE International Symposium on Robot and Human Interactive Communication, 2007, pp. 25-30. https://doi.org/10.1109/ROMAN.2007.4415048

[7] Khanday, O. M., Dadvandipour, S.: Convolutional Neural Networks and Impact of Filter Sizes on Image Classification, Multidiszciplináris Tudományok, VOL. 10, NO. 1, 2020, pp. 55-60. https://doi.org/10.35925/j.multi.2020.1.7

[8] Oniga, S., Vegh, J. and Orha, I.: Intelligent human-machine interface using hand gestures recognition. Cluj-Napoca, IEEE International Conference on Automation, Quality and Testing, Robotics, 2012, pp.559-563. https://doi.org/10.1109/AQTR.2012.6237773

[9] Kavarthapu, D. K.: Hand Gesture Sequence Recognition using Inertial Motion Units (IMUs). 4th IAPR Asian Conference on Pattern recognition, 2017, pp. 953-957. https://doi.org/10.1109/ACPR.2017.159 